\title{
RÉGIMEN URBANO: DE LA RUTA INICIAL A SUS BIFURCACIONES
}

URBAN REGIME: FROM THE ROOTS TO ITS FORKS

\section{Mario Bassols Ricárdez*}

\section{RESUMEN}

El texto expone los fundamentos bajo los cuales se construyó la propuesta central de la teoría del régimen urbano, en el marco de estudio de los gobiernos locales. Sintetiza algunas críticas importantes a sus planteamientos, así como formula preguntas en torno a la agenda de investigación urbana y las capacidades de gobierno desde la perspectiva analítica original. En los actuales estudios sobre la ciudad latinoamericana, una forma de empujar la agenda podría ser pensar el régimen urbano bajo una perspectiva relacional, perfilada desde el Sur. Ello implica abordar el análisis de sus peculiaridades espaciales, sus modos de gobernar y los problemas sociales que la aquejan.

Palabras clave: Régimen urbano, ciudad, gobierno local, agenda, poder.

\section{ABSTRACT}

This article casts a light over the main theory of urban regime analysis, in the local government studies field. It synthesizes important critiques to its fundamentals, and formulates questions around the urban research agenda and the governments capabilities, from its original perspective. Inside the current latin-american urban studies, a way to push the agenda, could be thinking about urban regime under a relational view, South profiled. This would implies diving into the analysis of its spatial peculiarities, its governing methods and the social problems that affect it. Keywords: Urban regime, city, local government, agenda, power.

Recibido 3I de agosto de 2020 y aceptado 30 de abril de 2021

* Profesor-Investigador, Departamento de Sociología, Universidad Autónoma Metropolitana Unidad Iztapalapa.<marbamex@gmail.com>. 


\section{LA PLATAFORMA DE INVESTIGACIÓN}

A 1 concluir la década de los ochenta del siglo pasado apareció una obra no, por su peculiar enfoque sobre ciudad, gobierno y poder. Su trabajo se insertó de lleno en el debate contemporáneo sobre las formas de abordar la ciudad desde las ciencias sociales. En Estados Unidos el impacto que tuvo la obra (Stone, 1989) fue suficiente para generar una creciente ola de estudios bajo esta línea durante más de dos décadas. En dicho libro se presentaba y desmenuzaba el concepto de régimen urbano. El mismo es explicado a partir de una ciudad que se va a convertir en su laboratorio de análisis, en el observatorio constante de sus transformaciones y en el semillero de las controversias surgidas posteriormente.

Su propósito central era concentrarse en las condiciones bajo las cuales surgían coaliciones políticas duraderas, con manifiestos públicos y acompañadas por funciones y tareas del aparato estatal, particularmente en la esfera de los gobiernos locales. Su propuesta de análisis se insertó en el debate sobre el urbanismo de fines del siglo xx, aun cuando durante muchos años fue una referencia constreñida a los estudios de política urbana en Estados Unidos. Fue considerado entonces como una perspectiva de análisis relevante, pero que se sumaba a otras ya desarrolladas de tiempo atrás sobre gobierno, élites y coaliciones políticas (Dahl, I96I; Pahl, I965; Peterson, I98I; Mollenkopf, 1983). El trabajo de largo aliento emprendido por Stone (I993; 200I; 2005; 2008; 20I5) ha sido continuo y en razón a su impacto ha tenido diferentes respuestas en la comunidad de estudiosos.

¿En qué consiste su fórmula para entender el proceso de «gestión urbana» en el capitalismo de fines del siglo xx? Bien, ese es un punto de arranque sobre lo cual cabe hacer una necesaria acotación, antes de proceder a explicarla brevemente. Se trata de una interpretación de la ciudad capitalista en su transición hacia una economía y una política de corte básicamente neoliberal, abierta al mercado global y bajo nuevos patrones de trabajo.

La fórmula de Stone es en principio sencilla: un régimen urbano es un típico proceso en el que se producen los arreglos informales por medio de los cuales el aparato público y los intereses privados se coordinan para hacer posible la toma de decisiones efectiva (traducción mía de Stone, 1989: 6). Es 
preciso contar con: (a) un marco institucional soporte de tales decisiones; (b) una cooperación de los diversos participantes (mediante incentivos selectivos), y (c) una agenda comprometida para llevar a efecto los proyectos sustantivos. En el contexto de las ciudades bajo las cuales desarrolla su análisis, prevalecen en principio dos cuestiones: (a) un sentido históricamente asentado de la libertad individual donde el dominio de lo privado prevalece por sobre lo público, y (b) una fórmula basada en coaliciones políticas (si bien asociadas frecuentemente a los designios de uno de los dos partidos dominantes en el escenario estadounidense) que explican la permanencia en el poder de un grupo determinado en el gobierno de la ciudad.

Se valora sobre todo un estudio de aquellos que cooperan en esa tarea y cómo esa cooperación se logra a través de sectores institucionales de la vida comunitaria. De esta manera, se abre la distinción entre diversas tipologías de régimen urbano, de acuerdo con los componentes de lo que Stone denomina «la producción social del poder».

Finalmente, el estudio de los regímenes urbanos debe asumirse a partir de un enfoque dinámico por medio del cual se ponen en tensión las tendencias que optan por el cambio o por la continuidad. De esta manera, se asume que un modelo de gobernabilidad asentado en el marco de un régimen urbano no es inmutable en el tiempo y en el espacio, sino que se encuentra en proceso de cambio (Stone, 200I). En seguida se presentan en forma sintética los ejes centrales de la teoría del régimen urbano.

El punto de partida de la explicación de Stone es que los gobiernos locales o gobierno de la ciudad no poseen por sí mismos la capacidad suficiente para gobernar. Por esa razón, deben recurrir a coaliciones políticas en las cuales hay grupos privados dominantes que proporcionan el soporte estratégico del equipo de gobierno. Tal coalición se constituye como un régimen, el cual, sin embargo, tiene la característica adicional de conformarse bajo un largo periodo, durante el cual se comparten e intercambian recursos. No se trata de un orden jerárquico de poder, sino que se construye con la colaboración mutua, aun cuando se susciten diferencias y desacuerdos. En el transcurso del proceso se construye una agenda de largo plazo que expresa un conjunto de objetivos e intenciones preferentes, cuyo valor es proporcionar una cierta cohesión al régimen urbano. No se basa entonces en un episódico proceso electoral en donde se producen coaliciones, frágiles o fuertes, pero tempora- 
les. Por sí mismas, carecen de la capacidad suficiente para movilizar recursos de forma sostenida.

De esa manera, Stone insiste en poner la atención en la «capacidad de gobernar». De aquí proviene su característica diferencia entre power to y power over (en inglés). Power to refiere al poder para "hacer cosas», mientras que power over indica, más bien, un sentido de dominación a la manera del pluralismo político o bien del marxismo. Es en este punto donde debe señalarse un enfoque que pone énfasis en las instituciones, pero sobre todo en las herramientas de análisis que ofrezcan elementos explicativos relacionados con la estabilidad. Así, los arreglos institucionales, las coaliciones y los recursos disponibles (ofertados en el mercado político) constituyen los componentes clave que lo llevan a diferenciarse de otras posturas del análisis urbano, como el de la teoría de las coaliciones en el poder local (en Molotch, 1976; citado por Domhoff, 20I7). En adición a ello, Stone se inclina más por el análisis institucional, que por la manera en que se ejerce el poder y hacia dónde se dirige este.

Ante la pregunta de "Who governs?» planteada décadas atrás por Robert Dahl, se podría responder que el foco de atención se centra en el proceso interno de arreglos informales que pone a funcionar la maquinaria de gobierno, con vistas a su permanencia en el largo plazo. De una manera simplificada es el buscar una explicación acerca de cómo es posible lograr la sustentabilidad política del gobierno urbano. He aquí un tema crítico que requiere poner debida atención para buscar posibles contradicciones en torno a una teoría del cambio.

\section{TIPOLOGÍA BÁSICA DE REGÍMENES URBANOS}

En este tenor, y conforme al análisis del autor, se obtienen cuatro tipos de regímenes urbanos:

I) De desarrollo

2) Progresista

3) Radical

4) Conservador 
I) El régimen de desarrollo tiene una agenda basada en la expansión y desarrollo de la ciudad, anclada en el "poder sistémico» que brinda la "comunidad de negocios local» (o grupos empresariales anclados regionalmente). Este tipo de régimen es clásico de las ciudades que crecieron durante la segunda posguerra, dentro de las cuales destaca Atlanta, pero también otras ciudades como Chicago o Detroit. En Atlanta, la élite empresarial dominó el escenario local durante varias décadas, pero debió lidiar con las emergentes demandas emanadas de otros grupos como el de los derechos civiles y las comunidades urbanas de base local, mediante «incentivos selectivos», aun cuando estuvo sometido a una intensa negociación.

2) El régimen progresista tiene un carácter más bien reactivo y su agenda se sitúa más allá de la expansión económica. Se trata de una fórmula basada en un discurso posmaterialista emergido de grupos de clases medias, artistas, universitarios e intelectuales de izquierda confrontados con el imaginario de una ciudad empresarial o ciudad del capital, según se le quiera ver. Sus preocupaciones se concentran en acciones de protección ambiental, derechos y libertades ciudadanas. También se elaboran proyectos de vivienda alternativos, enfocados a sectores vulnerables de la población, o bien de política social orientada a la lucha contra la segregación y la desigualdad social. Se asume que este tipo de régimen ha sido más exitoso en pequeñas y medianas ciudades, compactas y no disgregadas por la dispersión urbana característica de las metrópolis. El caso excepcional ha sido probablemente el observado en el régimen urbano de San Francisco, California, de la década de los noventa, cuando una coalición compuesta por ecologistas, conservacionistas y asociaciones pro lésbico-gays, dominó por largo tiempo una agenda que se opuso al modelo típico de ciudad pro-empresarial típica hasta entonces de la mencionada ciudad californiana (Bassols, 1994). Acaso un ejemplo más equilibrado y políticamente exitoso ha sido el de Portland en el estado de Washington, en los últimos años.

3) El tipo de régimen llamado radical es de rara presencia, pues está fincado en una amplia movilización de la sociedad urbana de masas, bajo la orientación de apoyar una ciudad basada en principios de equidad social, mayor acceso a empleo digno y búsqueda de oportunidades para los sectores 
vulnerables de la población. Las asociaciones civiles construyen proyectos financiados por fundaciones (nacionales o internacionales) y mantienen altos niveles de movilización social. El problema es su permanencia en largos periodos, por el necesario soporte financiero que suponen las acciones de tales grupos integrados a proyectos del gobierno local. Podría ser el caso de las llamadas por David Harvey «ciudades rebeldes», en el marco de una postura crítica frente a la política neoliberal (Londres en 20I9, como un caso).

4) Finalmente, se destaca el régimen conservador, esto es, aquel que no busca sino hacer que las cosas funcionen bajo la rutina del ejercicio burocrático del poder institucional. Probablemente, su presencia sea menos visible que las primeras dos mencionadas arriba, o hasta su existencia real puede ser cuestionable, en tanto que las ciudades no son inmutables en el tiempo. Las transformaciones del capitalismo se expresan en el espacio, aunque no de la misma forma en cada una de las ciudades. De existir, el régimen no es de tipo "puro" y más bien puede evolucionar a alguno de los otros regímenes, en tanto que la agenda también cambia con las transformaciones espaciales y socioculturales de una ciudad.

\section{ATLANTA: UN MODELO PARA (DES)ARMAR}

La obra de Stone es sólida como investigación histórica sobre los procesos de la política urbana en Atlanta en el siglo veinte. El análisis sobre las transformaciones socioespaciales de la ciudad, el papel de la élite blanca de tipo empresarial como grupo esencial de la política local, las crecientes tensiones raciales a las que se enfrentaron Atlanta y otras ciudades, en la década de los sesenta (Detroit, Chicago), y la emergencia de nuevos actores como los afrodescendientes (en términos demográficos y de acción política), son algunos de los temas bajo los cuales se analizan conceptos como el de producción social del poder, desigualdad social y eficiencia del gobierno local.

La existencia de un "régimen urbano» adquirió un sentido histórico, plenamente asentado en el escenario de la evolución urbana de las ciudades estadounidenses de la segunda posguerra. Se asume como una herramienta analítica poderosa para el examen de un cierto modo de gobernar el espacio urbano, dentro de periodos históricos de mediano plazo. El énfasis está dado 
en una agenda de gobierno (por medio de la cual se elaboran políticas públicas), la cual define así un cierto tipo de acuerdos basados en los problemas implícitos de la cooperación público-privada. Así, pueden distinguirse agendas del tipo: mantenimiento de la ciudad o bien conservadoras; las del tipo pro crecimiento económico, o aquellas orientadas hacia el fortalecimiento de grupos sociales de clases medias o populares en la economía de la ciudad.

El laboratorio «ideal» de análisis fue sin duda Atlanta, una ciudad bajo una economía emergente, con un empresariado que ejercía una insoslayable presencia en los negocios de la ciudad (no solo por Coca Cola Company, sino también por empresas ligadas al transporte masivo urbano, el Metro y otros negocios relevantes) y en la política local. Era ya entonces una urbe georgiana que en el imaginario urbano poseía una cierta identidad a partir de los sucesos en los que se vio involucrado el estado en la Guerra de Secesión del siglo XIX (novelada por Margaret Mitchell en The Gone with the Wind).

Buena parte del estudio de Stone se centra en las acciones de una nueva generación de alcaldes impulsados por las minorías de aquella época, para incidir en ciertas pautas de la economía política de la ciudad (desde el ascenso de Maynard Jackson como alcalde en 1973). Ello en el contexto de tensiones y desacuerdos que suponía impulsar una agenda de cambio orientada a fortalecer la economía de nuevos grupos de clases medias, pero basada en una coalición birracial. Durante el periodo 1946 a 1988, estudiado por el autor, abundan la protesta social y la lucha por los derechos civiles (uno de cuyos principales líderes a nivel nacional en los años sesenta fue Martin Luther King), el ascenso de movimientos ciudadanos y los desafíos planteados al gobierno local ante los grandes proyectos de transformación espacial. Stone establece un cierto contraste en la dinámica de los movimientos ciudadanos en Atlanta entre la década de los setenta y la de los ochenta. Durante la primera, se logró consolidar una fuerza política que le dio soporte esencial a la agenda urbana emprendida por M. Jackson y le permitió obtener mayores márgenes de maniobra frente a las resistencias de la comunidad empresarial y sus proyectos prioritarios; durante la segunda, que coincide con el ascenso de Andrew Young como alcalde de Atlanta en 198I (y su reelección en 1985), las organizaciones civiles, si bien mantuvieron su presencia en el Consejo de la Ciudad y en la legislatura estatal, dejaron de formar parte de la coalición de gobierno. Es entonces cuando se replantean las relaciones de cooperación 
con la élite de negocios, preferentemente blanca. El alcalde Young definió su función como dirigente local en esos años, bajo el propósito de conceder espacios de poder a los blancos (logrados por el soporte del electorado que lo llevó al poder), a cambio de obtener concesiones económicas para la comunidad afrodescendiente. Stone reconoce que tal fórmula no logró la equidad social, aunque sí se esforzó por fortalecer nichos de oportunidad en el ámbito de los pequeños negocios. Un ejemplo muy señalado fue el caso de la construcción del nuevo aeropuerto de Atlanta (con una de las mejores infraestructuras a nivel nacional), en donde empresas relativamente pequeñas de dicha comunidad obtuvieron contratos preferenciales. Esto es, el régimen urbano generó espacios de oportunidad para ser ocupados particularmente por la clase media negra que vio potenciado así su nicho empresarial. El gobierno de coalición birracial no puede verse en el marco de una relación tersa y permanentemente fluida entre ambas partes, tampoco como una fórmula del tipo suma-cero, en donde unos ganan mientras los otros pierden indefectiblemente. Prevalece ante todo la cohesión, una política de cooperación basada en incentivos selectivos y la definición negociada de proyectos prioritarios (ver caps. 3 a 8 de Stone,1989: 23-159).

En este espacio de tiempo, Atlanta se convirtió en un modelo de ciudad empresarial basada en una economía de servicios. No obstante, y a contrapelo, cuenta con más de 200 asociaciones y organizaciones ciudadanas y vecinales de carácter no proselitista y en pleno crecimiento económico dentro de las ciudades de Estados Unidos. Un modelo difícil de replicar, lo admite Stone, pero sin duda ninguna ciudad es replicable en esencia. Cada una tiene su singularidad y su marca. He aquí la Atlanta que va del periodo de la segunda posguerra a los ańos posteriores a los atentados del in de septiembre de 200I, cuando se reafirma el paradigma de seguridad nacional; igualmente están los cambios profundos en la economía mundial y la era posindustrial, bajo el anclaje de la globalización y dentro de un capitalismo heterodiverso.

Ha sido tal el reconocimiento de su obra que en un evento público celebrado en abril de 20I4, en la Universidad Estatal de Georgia, se esperaba la asistencia de Stone con gran interés por el público presente en el Auditorio. Además de estudiantes y académicos, había un contingente importante del público proveniente de organizaciones comunitarias de base de la ciudad de Atlanta. Ahí ofreció una conferencia sobre el antes y el ahora en los temas 
vitales de la política de la ciudad. Los activistas presentes en esa ocasión veían la obra que cumplía un cuarto de siglo como un texto que debería leerse por todos los involucrados en la praxis de la justicia social en Atlanta y le pedían una mayor reflexión sobre las lecciones que deberían extraerse del análisis de cara a los problemas de la desigualdad socioespacial en el siglo XXI (Hankins, 20I5: 156).

\section{LA OBRA Y SU CRÍTICA: UN ESBOZO DE SÍNTESIS}

En 2014 se cumplieron 25 años de la publicación del libro de Stone Regime Politics. La revista Urban Affairs Review publicó al año siguiente un conjunto de trabajos destinados a evaluar el impacto de dicha obra en el campo de la teoría y en sus ramificaciones ulteriores, así como enfatizar en algunas de sus principales valoraciones críticas. Un momento singular sin duda para el principal impulsor y defensor del concepto.

Algunos de sus principales resultados se pueden sintetizar en los trabajos presentados por Joel Rast (University of Wisconsin-Milwaukee), Michael Jones-Correa and Diane Wong (Cornell Univeristy, Ithaca, NY), Katherine Hankins (Georgia State University, Atlanta) y por el mismo Clarence Stone (George Washington University, Washington, DC). El debate, empero, ya existía y había arrojado múltiples trabajos de carácter crítico o propositivo sobre la obra de Stone, sobre todo en y desde los países anglosajones (entre estos, véase Lauria, 1997; Mossberger y Stoker, 200I; Davis, 2002; Guarneros, 2006). En México, y desde mediados de la década de 2000 , un pequeño colectivo de investigación llevó a efecto un proyecto basado en una primera revisión de los postulados centrales y las propuestas teórico-metodológicas centradas en dicha obra. El trabajo concluyó con la publicación de un libro, en torno a la metrópoli de Monterrey y cuatro de sus municipios centrales, más adelante comentado (Bassols: 2006).

En relación con un balance crítico fortalecido sobre el enfoque de régimen urbano, es importante establecer una comparación entre lo que se discutía a principios de los ańos noventa, las prioridades en la política urbana de entonces y las salidas conceptuales que Stone fue presentando en sus trabajos posteriores a 1989 (Rast, 2015). 
Por un lado, está el tema de las nuevas estrategias de cooperación público-privada en grandes ciudades, que dista de ser la dibujada por Stone al finalizar la década de los ochenta. En ese sentido, la crítica al régimen urbano en torno a su carácter localista cobra un particular sentido. Rast indica que el giro hacia la región es la tónica que priva en el marco de los procesos de negociación-cooperación. El autor se pregunta si dicho enfoque es capaz de aceptar esta estrategia espacial en el esquema de un régimen urbano.

El concepto de régimen urbano podría verse como una visión elitista sobre el poder, puesto que solo algunos grupos de la sociedad tienen la capacidad para movilizar recursos (financieros, políticos, institucionales) y así llevar adelante las agendas. Lo relevante, empero, es la combinación del análisis económico con la política local. Así,

[...] la narrativa de la Atlanta de los años 1980 trata de la historia de una ciudad que en la visión de Stone, gestionó con éxito la transición económica y política en virtud de la intervención de una coalición de élites económicas y políticas (Jones-Correa y Wong, 2015: 162). ${ }^{\mathrm{I}}$

En la revisión que hace un crítico de las teorías sobre el poder urbano en los Estados Unidos, la existencia de regímenes urbanos no parece tan común en la práctica, sino más bien se trata de casos excepcionales, entre los cuales se destaca Atlanta (Domhoff, 202I). Lo cierto es que la ciudad, como máquina de crecimiento, ya había sido estudiada bajo la óptica de la teoría de las coaliciones, pero desde el enfoque de régimen el punto que los diferencia es el peso otorgado por Stone al poder sistémico, y la fuerza que emana de las élites urbanas, dentro de una interpretación de comportamiento racional de los actores. Empero, cabe señalar las diferencias entre ciudades como Atlanta y Detroit, pues es en esta última en donde el poder sistémico se desarticuló con el declive de la ciudad, basada en la industria del automóvil y la ausencia de una agenda alternativa que previera opciones de amortiguamiento a raíz de la caída en vertical de su economía local, hasta su bancarrota financiera durante la segunda administración del presidente Obama.

${ }^{\mathrm{I}}$ Nota: Todas las traducciones son del autor. 
Con estos elementos presentes, podemos entender mejor el debate en torno al problema del cambio, así como las salidas ofrecidas por Stone bajo el concepto de «American Political Development» (APD). Vale la pena destacarlo, pues con dicho concepto se busca reafirmar los postulados centrales de la teoría del régimen urbano (en forma simplificada, se puede entender como teoría del régimen).

Rast encuentra que no es posible hablar de un sistema político integrado y coherente en todos sus niveles o ámbitos, como se supone en el enfoque de régimen, sino de múltiples perfiles u órdenes políticos construidos en diferentes momentos históricos, cada uno dirigido hacia diferentes propósitos. La noción de equilibrio implícita en las propuestas de Stone es cuestionada, pues ahora se debe poner énfasis en el desorden y en las posibles fricciones que suponen los acuerdos gubernamentales. Así, la teoría del régimen urbano enfatiza los acuerdos informales en el arreglo político, sin poner suficiente atención en las instituciones. En otras palabras, si el cambio es finalmente lo que tiene que ser explicado en la construcción de una agenda, los estudios sobre el tema no deben descuidar las texturas formales del orden institucional.

Como se ha visto, la teoría del régimen construida bajo los cimientos de Regime Politics y sus subsecuentes trabajos, parte de un patrón estable de cooperación a lo largo de un amplio periodo. En ese sentido, la dinámica implícita del régimen se refuerza constantemente y por sí misma. Esto supone que, en efecto, sea posible lograr la estabilidad y el equilibrio (siempre en tensión) por encima de las tendencias centrífugas de cambio. Y no es que Stone ignore esta posibilidad en el desarrollo histórico de una ciudad, sino que, explicadas así las cosas, cabe preguntarse cómo es que surgen las rupturas. $\mathrm{O}$ bien cómo esas fisuras que se abren con el tiempo pueden conducir a nuevos arreglos.

La crítica central a Stone se basa precisamente en la siguiente afirmación:

No se identifican aquellos mecanismos de cambio endógeno al sistema que hagan posible transformaciones sustantivas en los acuerdos de gobierno. Se trata en otras palabras, de un problema entre estructura y agencia. De cómo no sólo los acuerdos informales en el espacio de la acción política deciden la agenda, sino que la presencia de factores de mayor peso (en el orden económico, social y cultural) en las políticas 
urbanas, forman la baza de los acuerdos. Las estructuras de poder existen, pero no controlan todo (Rast, 2015: 142).

En cierta manera, los grupos decisionales no se involucran en todas las áreas de interés, por lo que algunas de ellas quedan «al margen» y pueden ser retomadas por otros grupos fuera de la élite de poder. Este asunto relacionado con la dificultad de reconocer otros puntos de apoyo dentro de los márgenes de la política urbana será una de las críticas emergentes a la teoría del régimen. No se reconocen márgenes de acción importantes, más allá de la cuña lograda por esa combinación exitosa entre las élites para lograr la transformación de una ciudad y mantener agendas de largo plazo. ¿Pero para quién y a qué costo se logran?

De nuevo el ejemplo de Atlanta ilustra esta cuestión: los esfuerzos emprendidos para limpiar las zonas de mayor deterioro urbano (en aras de una refuncionalización urbana) implicaron efectos colaterales mayores de los previstos. Además, cabe considerar las distintas respuestas que en ese margen de acción han emprendido distintas asociaciones de vecinos en relación con el ritmo y la tónica del desarrollo urbano preferencial. Esto es, aquel desarrollo que domina, en efecto, la agenda pública de la ciudad.

De lo anterior se puede colegir un problema en la estructura, esto es, en las condicionantes estructurales, que si bien dan margen a la acción de los agentes dominantes en la política local, también restringen las posibilidades del ejercicio político, tanto como las consecuentes transformaciones institucionales. Y con todo ello habría que reconocer el papel creciente de las minorías afrodescendientes en el juego de la política local, como sucedió en la ciudad de Chicago en la era de Harold Washington entre 1983 y I989 (Bassols, 1994). De esta forma, una lección derivada del estudio de la política local urbana en ese país es que, si bien la asociación público-privada orientada a los grandes negocios del capital se mantiene, ahora debe ser capaz de reconocer que su poder se ha disminuido. Son cada vez más voces en el concierto de la política local (y nacional), nuevas demandas, viejos reclamos, emergencias de liderazgos por parte de ciertas minorías y, en ese sentido, un mayor pluralismo en la lucha por el poder.

Las decisiones en materia de política pública serán foco de atención de grupos, a partir de sus propias trayectorias culturales y experiencias colecti- 
vas. Basta con revisar las decenas de páginas web de asociaciones ciudadanas en Atlanta, desde cuya plataforma se discuten y evalúan los proyectos urbanos de base territorial, emanados del gobierno local o de iniciativas empresariales a diferentes escalas de intervención espacial.

Para este propósito, es ilustrativo el análisis de Jones-Correa y Wong en torno a las posibilidades de éxito que tienen los grupos que actúan sobre los márgenes de la agenda dominante. Utilizan como ejemplos los casos de las siguientes organizaciones: (I) «Los Sures United Housing Development Fund», en relación con el problema de la vivienda de bajo costo en Nueva York, la cual ha logrado construir una plataforma de resistencia ciudadana y de solidaridad para enfrentar desde el espacio local el incremento catastrófico del precio de la vivienda en el epicentro de la ciudad global. Su posición como intermediario entre los residentes locales y el gobierno de la ciudad le ha conferido un relevante papel en la política de la ciudad, así como ser una plataforma para la movilización electoral. Su existencia data de I972; (2) «Koreatown Immigrant Workers Alliance», fundada en 1992 para defender los derechos laborales de koreanos y latinos en la zona de Koreatown en Los Ángeles. Se ha enfocado en la organización de trabajadores ligados al sector servicios, particularmente quienes trabajan en restaurantes y supermercados. En 2007 dicha organización ganó un pleito judicial a la compañía Assi Super, Inc., por un millón 475 mil dólares, por discriminación racial hacia trabajadores latinos y por no haber pagado tiempos extra a sus empleados. En los bordes de la política local dominante se mueve un amplio espectro de organizaciones de base comunitaria que llevan a cabo labores de defensa (advocating) para tales grupos de trabajadores y, por ello, le imprimen una presión inusitada tanto a las organizaciones laborales reconocidas formalmente, como al mercado laboral; (3) «Grace Lee Boggs Center», cuya acción se centra en recuperar los espacios escolares perdidos en Detroit durante su declive económico (según estadísticas nacionales, 1929 escuelas públicas fueron cerradas tan solo entre 2010 y 20II). Surge para tal efecto una coalición para la justicia educacional, cuyo tema lo inserta dentro de la agenda de los derechos civiles. Bien es sabido que Detroit ha afrontado una larga crisis en la era posindustrial (y de la ciudad del automóvil). Las escenas del filme Gran Torino de Clint Eastwood dan una idea de este desconcierto social. La 
asociación de Boggs Center pone el acento en el proceso educativo que tiene lugar fuera del salón de clases y que contribuye a generar lazos comunitarios.

Por medio de un trabajo de concienciación entre habitantes afectados (por lo general, los de mayor fragilidad económica que viven en barrios negros depauperados o semivacíos), han logrado movilizar a comunidades para involucrarse más en la educación pública dentro de los distritos escolares. $\mathrm{Y}$ aunque no todos los esfuerzos de tales organizaciones son fructíferos, su permanencia en la acción ciudadana local redimensiona los esfuerzos que desde el vecindario urbano se realizan en torno a la política educativa (Jones-Correa y Wong, 2015: 167).

\section{DE LA AGENDA COHERENTE A LA DIFUSA EN EL GOBIERNO LOCAL}

Para Stone, pese a los ajustes que ha implicado el paso de una agenda coherente a una difusa en los modos de gobernar la ciudad, la misma sigue siendo definida por las grandes líneas marcadas por las élites. No obstante, reconoce que el interjuego entre actores locales y régimen se presenta en el marco de una desigualdad estructural, que en resumidas cuentas conduce a favorecer a ciertos grupos con recursos y dejar al margen a los que no lo tienen.

Dada esta postura, a todas luces inamovible de Stone, otros autores (Jones-Correa y Wong, 2015: I68) apuestan por una diversidad de actores en la arena de la política local. Esta debe verse en un sentido más complejo y multidireccional, al existir grupos activos capaces de movilizar recursos y rediscutir agendas por largos periodos. Así se puede tener una mejor lectura de los esfuerzos emprendidos desde nuevos frentes de la vida cívica: las minorías étnicas y raciales, inmigrantes, jóvenes y todos aquellos que en términos generales enfrentan una escasez de recursos económicos.

En síntesis, se trata de buscar explicaciones más complejas del cambio, que incorporen variables endógenas y exógenas al modelo urbano implementado. Se pone de relieve el «poder comunitario de base» presente a lo largo del tiempo y en distintos tonos e intensidades, unas veces actuando en consonancia con el arreglo político dominante, pero en otras, sobre todo, en desafío a las instituciones formales de gobierno. El delicado equilibrio entre élites blancas, clases medias y organizaciones comunitarias logrado en 
Atlanta durante el periodo analizado por Stone, no es ciertamente la única forma de construir una agenda basada en prioridades de expansión económica y estabilidad. Lo valiosos del caso de Atlanta es que, pese a tratarse de un «régimen de excepción» (por su naturaleza pocas veces replicable), se pueden extraer múltiples enseñanzas sobre la forma de lograr acuerdos en política pública.

\section{UNA CRÍTICA DESDE EL REGULACIONISMO $Y$ «EL ENTIERRO CON HONORES»}

Otra de las lecturas en torno a la obra de Stone parte de un enfoque regulacionista, en donde se reexamina el régimen urbano desde parámetros en donde, además de los procesos generados desde la economía, el espacio y las estrategias de los actores también cuentan. Es importante mencionar que el modelo de producción social del poder puede asumir varias lecturas, pero la relación entre recursos y agenda, como punto de partida, parece una regla inamovible. Los actores urbanos que dominan la escena son, en efecto, quienes moldean las prioridades del desarrollo.

El análisis del caso de Atlanta puede ser visto como complemento a la escuela del regulacionismo (Aglietta, 1999) en el siguiente sentido: si lo que Stone propone está ligado a la capacidad de agencia de ciertos actores en el marco de las transformaciones de una ciudad, entonces se entiende que tiempo y espacio moldean la estructura en que se reproduce el modelo en cuestión. El enfoque en que se basa la autora es aquel que pondera las formas particulares en que el capitalismo se las arregla con sus propias crisis, considerando que dicho manejo se produce a través de diferentes circunstancias culturales e institucionales. El capitalismo se produce y reproduce en sus crisis estructurales, pero su estabilidad (de suyo frágil) atraviesa por instancias que tienden a regularla, como una organización institucional determinada, formas y normas sociales de vida, procesos políticos constitutivos del régimen de gobierno o culturas políticas dentro de una sociedad determinada.

Más allá de las limitaciones intrínsecas del régimen urbano, es preciso redimensionarlo hacia una visión de mayor calado sobre el cambio político urbano. El señalado propósito de evitar caer en las trampas del localismo señaladas por Gerry Stoker (1995) lleva a introducir el problema de las esca- 
las en la política urbana. Así, los procesos globales también son parte de la experiencia de lo local, en tanto que las relaciones socioespaciales asumen múltiples formas y dimensiones (Henkins 20I5: I52).

Bajo este supuesto, es útil recurrir a la conceptualización de Edward Soja sobre la dialéctica socioespacial: la forma espacial no solo está moldeada por los procesos de producción social de la ciudad, sino que en sí misma es parte activa del modelo. $\mathrm{O}$ en una versión más compleja se concibe como una trialéctica del espacio: el percibido, el concebido y el vivido (Hernández, 2008: 92-93). Si atendemos a esta diferenciación, se encuentra una diversidad de actores en juego, con sus visiones y rasgos particulares de acción, representación y prácticas sociales en y sobre el espacio.

De lo anterior se desprende que tanto los artefactos instalados en la ciudad, su forma urbana, la infraestructura y los recursos disponibles, como los grupos sociales mismos que cotidianamente se movilizan, permean todos ellos la vida política local. Para Henkins (2015: 154), el análisis histórico de las transformaciones espaciales en Atlanta muestra claramente la manera en que en varias décadas el régimen urbano se esforzó por desalojar a los afroamericanos de sus viejas barriadas del centro, bajo los conocidos proyectos de «remodelación urbana», a fin de diluir su concentración de poder político. Este proceso puede ser examinado ahora en el largo plazo y constatar que el régimen urbano sigue presente en el proyecto de ciudad, lo cual para dicha autora tiene sus efectos consecuentes en el ámbito de la acción política.

La cuestión no consiste tan solo en definir la manera en que los regímenes urbanos existentes se producen bajo determinadas circunstancias histórico temporales (en donde el recurso al análisis regulacionista cuenta), sino finalmente cuáles son las consecuencias en el espacio derivadas de su implantación, así como los procesos de política producidos a su vez por dichos espacios.

Para ciertos críticos de la teoría del régimen urbano (Sapotichne et al., 2007), después de un periodo más o menos largo de dominio dentro de los estudios sobre política urbana es la hora de hacer el corte y empujar decorosamente la caja que la contenía hacia el panteón de los ilustres: «un entierro con honores». Pero ¿qué pasa entonces con los modelos de interpretación que se constituyen como paradigmas? Para Thomas Kuhn, los paradigmas no desaparecen solo porque una comunidad de científicos ha comenzado 
a perder la confianza depositada en alguno de ellos. Es condición necesaria que emerja un nuevo cuerpo teórico capaz de rivalizar y ser suficientemente persuasivo para asestarle un duro golpe al orden de ideas prevaleciente. $\mathrm{Si}$ esto aplica sobre todo a las ciencias duras, es posible pensar que en las ciencias sociales algo similar ocurre en la comunidad de estudios. Así, no es que haya aparecido un nuevo paradigma que ponga en la sombra al cuerpo conceptual propuesto y liderado por Clarence Stone, sino que las alternativas de explicación son más diversas y, por tanto, más fragmentadas y parciales (Rast, 2015: 146).

Así, por ejemplo, Katherine Hankins (2015: 157), desde una perspectiva geográfica, reconoce sus fortalezas al ser una lente poderosa para examinar la política urbana. El punto de tensión radica en la necesidad de observar nuevos escenarios en torno a lo que un régimen urbano progresista podría ser y cómo esto podría lograrse en escenarios alternos al de Atlanta o al de las ciudades estadounidenses. Se dibuja una dialéctica socioespacial capaz de entender el ejercicio de la política local, en el marco de una agenda de investigación con miras hacia temas globales relevantes, tales como la pobreza y el desarrollo desigual.

\section{EXAMEN INTROSPECTIVO}

El debate sobre la teoría del régimen ha sido largo y con múltiples ramificaciones. Ha permitido clarificar cuáles pueden ser los posibles usos y sentidos de la propuesta. En sí mismo, el término mismo ha sido «incómodo» como el propio Stone lo reconoce. Él mismo propone pasar de una teoría del régimen a un "análisis del régimen urbano». El texto es muy sugerente, pues resume el aprendizaje obtenido por un autor sobre su propia obra, considerando los aportes desde otras perspectivas, las diversas críticas a su obra y las respuestas que este ha ofrecido en diversos momentos. No obstante, por la forma en que desarrolla sus argumentos, el diestro manejo de autores (todos ellos dentro de la literatura anglosajona) y las conclusiones de su trabajo, permiten afirmar que constituye una actualización de sus propuestas y una reafirmación de sus postulados básicos.

El apartado más amplio se dedica a su libro de 1989. Un segundo apartado, más corto, intenta ubicar el modelo de Atlanta en un esquema de 
interpretación del capitalismo y el papel de las ideas en el cambio político. Finalmente, una tercera parte se orienta a reflexionar sobre los procesos políticos urbanos y los modos de gobernar ciudades, en donde presenta algunas ideas a manera de conclusión.

Es preciso poner atención sobre los puntos fuertes y debilidades que, a la luz de los años, presenta su original y polémica obra. Stone distingue dos componentes centrales: la dinámica interna suscitada en los procesos de acuerdos gubernamentales para lograr definir prioridades de la agenda, y el análisis del contexto en que se producen esos acuerdos en el largo plazo.

En esta tesitura, las modificaciones en el formato gubernamental, lejos de ser altamente cohesivo como en el pasado, son ahora difusas y con una agenda menos clara en sus propósitos públicos. Por otro lado, deben ponderarse las ventajas de un análisis de los componentes contextuales, en tanto que brinda una dimensión distinta del cambio político. El caso de Atlanta resalta por sus propias características, si no únicas, sí lo suficientemente relevantes como para definir a su régimen urbano a partir de una coalición gobernante de tipo birracial, construida desde el fin de la ir Guerra Mundial. En tal caso, el estudio se concibe como un instrumento heurístico para "capturar» la forma en que tienen efecto los procesos de política, en muchas ciudades durante un periodo específico. Lo anterior nos parece de lo más relevante y es necesario indagarlo en sus contextos particulares de análisis urbano.

Pese a las críticas, Stone reafirma su «ley de hierro» o corazón del análisis: aquel en el que para hacer factibles los acuerdos públicos, los recursos disponibles deben ser acordes a la agenda. Para observar una agenda sustancial y sostenida, se requiere entonces una coalición estable, sin la cual no es posible contar con tales recursos. Por cierto, reconoce la posibilidad de que asociaciones no gubernamentales pueden ser parte de la coalición de gobierno.

El acercamiento a un esquema de redes de cooperación cívica es acaso más palpable ahora, dentro del esquema más conocido de gobernanza (Porras, 2016). Si estos elementos se alteran significativamente en el tiempo, es altamente probable que el régimen se disuelva o en su caso se transforme. Esto es, tiene la capacidad de: (a) ajustarse y adaptarse, o (b) desaparecer.

No es en vano insistir en que el régimen urbano no es algo cerrado o indescifrable, aunque aparenta ser una especie de cuña de poder infranqueable. El periodo de gobierno del primer alcalde afroestadounidense en Atlanta lo 
demuestra, pues si bien se mantuvo una agenda de crecimiento económico bajo el eje capitalista, al mismo tiempo fue capaz de romper con la vida cívica que caracterizaba a la ciudad en los años setenta, en donde prevalecía la separación racial y la confrontación cotidiana. Se trabajó en una política que tendió puentes para una mayor interacción cívica y empresarial. Nuevos modos de cooperación que hicieron de Atlanta la Mecca del afrodescendiente (Stone la llama The Black Mecca), por su demostrada capacidad de adaptarse al cambio racial, a diferencia de lo que ocurrió con Chicago, Oakland y otras ciudades donde se escenificaron importantes procesos de incorporación política de minorías. En ese sentido, Atlanta es vista a partir de prácticas de «buen gobierno» local, considerando una escasa plataforma de acción de los partidos políticos. Y esto es algo que marca una clara diferencia con la política urbana en ciudades mexicanas del siglo presente: la hegemonía del partido en el poder por sobre las organizaciones civiles y ciudadanas locales.

Stone retoma la perspectiva del «Desarrollo Político Americano» para asentar que el cambio es algo inherente a la política como tal. Lo central es entonces cómo el cambio y el orden están interrelacionados (Stone, 2015: I05). Una periodización de tales transformaciones se hace necesaria y Stone ubica la mayor parte del periodo estudiado como uno fundado en el re-desarrollo. En otras palabras, el impulso a la revitalización económica de la ciudad. En las décadas posteriores a los años ochenta, las condiciones han cambiado en términos generales: el patrón espacial de los usos del suelo, el declive de la ciudad industrial y el énfasis en una ciudad de servicios basada en la nueva era de la información, llevan a un cambio en las prioridades de la agenda.

A las críticas sobre un exceso de localismo, en el que se asume que las ciudades están ampliamente autocontenidas, o bien que sus procesos locales se explican por sí mismos, Stone ofrece una respuesta cercana al regulacionismo. Insiste en el papel mediador de los arreglos informales del gobierno local. Ellos permean los procesos que desde la economía global impactan en las comunidades locales. Reconoce la importancia del localismo en la historia del Estado moderno en su país de origen. Así pues, el cambio político no sigue una simple lógica que se apega estrictamente al desarrollo capitalista, aún en su fase neoliberal. Basado en Charles Tilly (1984) y otros, el cambio 
político está históricamente constituido y de esta forma consiste en una sucesión de configuraciones.

Si ya no puede sostenerse como tal la idea de que el régimen urbano debe basarse en una coalición coherente y estable en tiempo y espacio, el teórico de Atlanta se inclina por pensar en un proceso multivinculado a la hora de gobernar una ciudad y ello dentro de una amplia perspectiva de las transformaciones socioeconómicas ocurridas en la ciudad. Se han producido cambios en la estructura del transporte y de la red de comunicaciones (del ferrocarril y el tranvía a una era basada en el automóvil y últimamente a nuevas formas de movilidad urbana e interconectividad), en la manera en que la sociedad se inserta en la ciudad informacional, o bien se suscita la emergencia de una clase creativa. Todos ellos son factores que, junto al uso del suelo, reconfiguran las agendas. Habría que considerar, además, el declive de una postura basada en la planeación estratégica de largo plazo, para sustituirla por una centrada en los beneficios de corto plazo en la economía global. Asimismo, varias ciudades han sido atravesadas por procesos migratorios de larga data. Se convierten en un factor demográfico cuyos cambios impactan en la ocupación del espacio y en la defensa de valores y derechos, particularmente por una empoderada clase media afroestadounidense y minorías emergentes.

Ello, junto a los procesos de multiculturalidad y etnicidad de la sociedad presente, constituyen uno de los mayores desafíos para sostener la cohesión social. Sin duda, el nuevo paradigma está ligado a patrones del tipo «seguridad nacional», lucha contra el terrorismo y las acciones que constituyen actos de desafío al orden social prevaleciente.

\section{PRIMERAS CONCLUSIONES}

Una conclusión relevante de su estudio es que al correr el siglo XxI, con todo y la efervescencia social de los grupos subalternos de bajos recursos, ellos siguen permaneciendo como "políticamente marginales», aunque menos dóciles que en la era previa del régimen urbano. La acción local es, no obstante, el principal determinante de todas las formas que toma la reconstrucción social. Gobernar una ciudad se convierte en un proceso más fluido que en 
el pasado. Todo ello bajo el reconocimiento de contextos donde priva la desigualdad estructural y la escasez de recursos.

Es en este punto en donde cobra sentido el debate sobre si la desigualdad prevaleciente bajo el capitalismo es no solo inevitable, sino simplemente es la palabra final acerca de las explicaciones sobre la vida moderna. Tal sería el caso de la pobreza urbana y de las estrategias para su disminución, ya que según Stone deben ser examinadas a través de mediaciones. En consecuencia, la simple dicotomía entre poder y pobreza es cuestionable tanto en países de alto desarrollo económico, como en las ciudades latinoamericanas. Existen bordes que pueden ser maleables entre corrientes dominantes y marginales. Por otro lado, la injusticia racial y los derechos desiguales a la ciudad atraviesan las agendas tanto de las políticas locales en ciudades, como de la agenda federal. Son, pues, temas recurrentes que adquieren mayor relevancia en tiempos electorales, como fue el año 2020 en los Estados Unidos.

En términos de régimen urbano, se trataría de observar cuáles son las capacidades de gobierno para desafiar la exclusión y obtener recursos para combatirla. He aquí un problema de agenda: una capacidad para actuar, o power to, considerando las restricciones presupuestales presentes en la formulación de políticas públicas. Así está el dilema de hacia dónde, con qué recursos y en qué circunstancias se deberán aplicar determinadas políticas. Como señala el analista Richard Rose (Rose, 1998), «no hay política sin dolor» y en eso consiste parte del debate sobre cómo diseńar, implementar y evaluar políticas públicas en la era actual (Merino, 2013). Frente a las distintas posturas de análisis, se precisa una nueva convergencia que realice la comparación entre los rasgos de un periodo de tiempo con otro.

Si bien resulta menos complejo determinar el momento del declive de determinados acuerdos de gobierno, el desafío más relevante es, en este caso, establecer cuándo una era está cerca de llegar a su conclusión. De ahí la importancia de establecer periodizaciones claras de régimen. Esto puede ser útil a manera de observación metodológica para un análisis específico de ciudades y gobiernos locales.

Queda pendiente la confirmación de si la era del régimen urbano tal y como se le concibió ha llegado a su fin. En cualquier caso, el enfoque de régimen urbano se afanó en construir una explicación muy elaborada de cómo las ciudades son y pueden ser gobernadas. Ahora se ensayan nuevos 
proyectos bajo enfoques más amplios y pluridisciplinarios, algunos situados en explicaciones más complejas sobre los perfiles de la política local. Se puede discrepar con el mismo, pero otra cosa es ignorar sus aportaciones al estudio de la ciudad capitalista posindustrial.

\section{CAMINOS RECORRIDOS Y POSIBLES RUTAS}

Una mayor pluralidad teórica se distingue en el firmamento y ello tiene algunas implicaciones de mayor calado en cuanto a su alcance explicativo, a los presupuestos de los cuales parte y al conjunto de temas incorporados. Considérese, por ejemplo, el vertiginoso ascenso del modelo basado en un urbanismo neoliberal, el cual ha dejado atrás el legado keynesiano de organización del espacio urbano. Es decir, en el que fijaron sus posiciones las escuelas o corrientes dominantes en décadas atrás acerca de la ciudad capitalista y de sus beneficiarios más próximos (Forrest y Wissink, 20I7; Mayer, 20I7). Una de las discusiones más relevantes en los actuales tiempos consiste en replantear el paradigma de la ciudad capitalista de la posguerra, el cual se sustentó en un Estado provisor de bienes y servicios (aun desigualmente distribuidos). Los órganos del Estado pierden capacidad para regular los procesos de producción del hábitat en las ciudades. También pierden legitimidad política. En este mismo sentido, cobra fuerza una amplia discusión sobre el espacio público y el «derecho a la ciudad», así como las alternativas que emergen desde los gobiernos locales frente al urbanismo neoliberal o posfordista.

Se impone un ejercicio de reflexión sobre algunos ejes de análisis del poder urbano en grandes ciudades. La teoría del régimen nos empuja a desarrollar respuestas más detalladas a preguntas tales como: ¿de qué manera se construye la agenda en el corto y mediano plazo?, ¿cómo entender la continuidad y el cambio en el poder urbano y el gobierno de la ciudad?, ¿Cómo redimensionar, en las ciudades latinoamericanas, el contenido de la agenda urbana en contextos de expansión metropolitana?

En seguimiento a una línea temática iniciada a principios de I990, se emprendió entre 2002 y 2005 una investigación colectiva desde El Colegio de la Frontera Norte en su sede regional de Monterrey, México. Fue una experiencia en la que se tuvo por primera vez en nuestro país una confron- 
tación con tal enfoque. Ello tanto desde el punto de vista de sus principales fundamentos y sus pilares teóricos, como de los desafíos metodológicos que representaba llevar a cabo una investigación de campo, en el Área Metropolitana de Monterrey. Los primeros dos capítulos de la obra daban cuenta de una evaluación crítica de la teoría del régimen urbano y las lecturas que desde el continente europeo se tenían al respecto. Asimismo, se examinaban cuatro municipios del Monterrey metropolitano, muy distintos entre sí en su composición política y partidista (Monterrey, Garza García, Apodaca y García). En el tramo final se presentaba una interpretación de actores clave, tipificados desde su habitus propio, según las directrices del sociólogo Pierre Bourdieu (Bassols, 2006).

Como resultado principal del proyecto quedaron varias enseñanzas sobre las entrañas de la política urbana y la madeja de intereses y constricciones, que envuelve a las tareas de gobierno en ciudades y metrópolis desde el contexto nacional. Al aparecer la multicitada obra de Stone, México estaba enfrascado en una lucha por la transición democrática y la alternancia en el poder. Las reformas políticas se habían iniciado en la segunda mitad de la década de los setenta, pero tuvieron resultados más visibles años después: en gobiernos municipales primero, en los estados a partir de 1989 y en 2000 con el triunfo del PAN en la contienda electoral por la presidencia de la república. Los estudios sobre gobernabilidad y buen gobierno dominaron el escenario en la década de los noventa y principios del presente siglo. Con el cambio de siglo cobrarían fuerza los estudios basados tanto en los enfoques de la gobernanza, como en los de la acción pública.

Es en este espacio de tiempo en el que se inserta el mencionado trabajo sobre régimen urbano en Monterrey. Como se ha indicado, los trabajos de Stone experimentaron un bajo impacto en los estudios latinoamericanos y del Caribe. Sobre ello se ofrecen las siguientes explicaciones posibles.

La primera tiene que ver con una sociología del urbanismo o de los estudios urbanos. Es decir, las formas en que se institucionaliza la investigación urbana, sus ritmos y discontinuidades, las culturas académicas dominantes, el tipo de trabajo académico, etcétera. La segunda viene de la fórmula retomada por Emilio Duhau del antropólogo Lins Ribeiro: la combinación entre "cosmopolitismo periférico»y "provincianismo metropolitano», que aún es 
la tónica común en los estudios urbanos de nuestra subregión americana. Decía entonces:

Los investigadores latinoamericanos habitualmente nutrimos nuestras investigaciones con la producción proveniente tanto de nuestro propio país y región como la de la originada en otras regiones y, en tanto sean accesibles en lenguas que somos capaces de leer, como el inglés y el francés, por regla general, así como en nuestras propias lenguas, es decir, español y portugués. Y a la inversa, también por regla general, los investigadores formados en las tradiciones académicas de los principales países desarrollados, particularmente los estadounidenses, salvo cuando se especializan en otras regiones, ignoran olímpicamente la producción que dentro de su campo de investigación, es ajena a su propio mundo lingüístico-cultural (Duhau, 2006: I3).

Finalmente, la tercera explicación estriba en el modelo de organización socioespacial de las ciudades latinoamericanas y del Caribe, que no obstante reproducir estereotipos socioespaciales que tienden a semejarse a los de las ciudades de aquellos países (sobre todo, en México es visible la influencia del modelo estadounidense de ciudad), distan de ser similares.

Puesta la mirada en el escenario de las metrópolis contemporáneas latinoamericanas, las explicaciones pueden ser más complejas cuando un conglomerado urbano compuesto por varias ciudades y en ocasiones «archipiélagos urbanos» desconectados entre sí contiene varias agendas de gobierno, según la escala espacial nudo de la problemática, la incidencia territorial diversa de sus actores y los problemas del federalismo implícitos en la acción gubernamental. Muchas de estas mantuvieron una dinámica de expansión territorial, demográfica y de cambio económico intensos en las últimas tres décadas (hasta el año 2020). Por igual, y pese a sus limitaciones, la transición de una economía de base industrial a una de servicios, quiérase o no, se ha realizado.

El alcalde de una ciudad central (en capitales federales, por ejemplo) y sus órganos de gobierno local, pese a su fuerza en el contexto metropolitano, carecen de capacidades efectivas y muchas veces de voluntades políticas para lograr o imponer por sí mismos los acuerdos necesarios para lograr una 
agenda única. De hecho, lo que muchas veces sucede es que el alcalde se enfrenta a múltiples frentes en el cotidiano de la acción pública. No gestiona una sola agenda, sino varias, unas de mayor relevancia que otras, según la coyuntura y las áreas de interés en juego.

En una metrópoli, las agendas son contradictorias o disímiles y no se definen en un solo centro de poder. En ese sentido, el poder metropolitano es centrífugo y poroso, como la ciudad misma. Es difícil reconocer «actores metropolitanos» o cuyos intereses e influencia territorial se sitúen en la escala regional: hay superposición de poderes, órdenes de gobierno y grupos empresariales y de la sociedad civil, con lógicas de acción diversas. Eso ciertamente ocurre en todo contexto metropolitano, tanto en Atlanta, Nueva York, Los Ángeles, como en Buenos Aires, Bogotá o Ciudad de México. Acaso una diferencia central entre los gobiernos de ciudades norteamericanas (Canadá o Estados Unidos) y los de América Latina y el Caribe, radica en la fragilidad o fortaleza del poder local, según sea el caso. Los alcaldes de aquellas ciudades poseen márgenes de maniobra mayores para incidir en las agendas de gobierno, negociar acuerdos con empresarios, imponer ciertos límites o conceder incentivos selectivos a otros grupos sociales. Sin embargo, ejercicios de gestión innovadoras en su tiempo, como las del tipo de "presupuesto participativo» en Brasil, muestran la importancia de lograr acuerdos políticos en la discusión de programas gubernamentales.

Ciertamente, en la era del capitalismo global y sus transformaciones, las agendas pueden ser contradictorias: desde el orden de la economía trasnacional y de los derechos universales del hombre, hasta los de la política de barrio y de las comunidades locales en donde también se toman decisiones, bien de defensa a sus tradiciones o de oposición a los megaproyectos urbanos que tienden a destruir ese tejido social histórica y espacialmente construido. La conformación de ciudades difusas, con urbanizaciones discontinuas y territorialmente heterogéneas, pero integradas económicamente a través de complejas redes de infraestructura de comunicación, lleva a pensar en la formación de metapolis (García, 2016: I42). Como sea que se les caracterice, «las ciudades se mueven», los gobiernos locales existen a partir de una representación política determinada y la gestión urbana es continua, a pesar de lo contradictorias que puedan ser sus agendas. En consecuencia, el estudio 
de la política local en contextos megalopolitanos o aún metapolitanos sigue siendo un eje de análisis urbano relevante.

Queda claro que la teoría del régimen no estaba pensada para su encuadre analítico en las ciudades latinoamericanas. Hay que decir que tampoco se la estudió a fondo. Pero aun ahora el ejercicio no resulta en vano, cuando una rampante economía neoliberal exhibe todas sus fisuras y parece diluir la importancia de las agendas públicas locales y de la planeación territorial.

Así, trabajos como el realizado para Buenos Aires (Guevara, 20I5) en torno a los grupos de poder local y los proyectos urbanos dominantes, son muestra de ello. También pueden serlo como un ejemplo del interés que se mantiene sobre esta perspectiva de análisis urbano, pese a tener aún escasa incidencia en el conjunto de los trabajos sobre metrópolis latinoamericanas.

Un proyecto relevante en tal sentido es el que se propuso indagar sobre la existencia de distintos modelos de gobierno en las ciudades de América Latina. Ello, dado el peso que habían adquirido (desde fines del siglo xx y con el ascenso del urbanismo neoliberal en la región) los procesos basados en «la eficiencia económica, la racionalidad administrativa y la prestación de servicios» (Carrión y Ponce, 2015: 13). En el marco de las discusiones en eventos y reuniones de trabajo, la cuestión a dilucidar era obtener un conocimiento más detallado sobre cómo un proyecto de izquierda en la política local se expresaba en la gestión urbana de las ciudades latinoamericanas. En términos de la tipología propuesta por Stone, se podrían considerar rasgos del régimen progresista y del radical, para a su vez contrastarlos con los ejemplos analizados en dicho proyecto: Lima, Porto Alegre, Rosario, Montevideo, Bogotá, Quito, La Paz, Ciudad de México, Santiago (fuera de América Latina: Portland en Estados Unidos). Pero el llamado "giro a la izquierda» no es una tendencia fija y permanente. Se manifiesta en discontinuidades y es precisamente en las agendas de gobierno y el cambio en sus componentes partidistas y coaliciones políticas en donde se localizan sus diferencias y peculiariedades.

La obra señalada es una expresión del nivel alcanzado en la investigación urbana en América Latina en la línea de análisis sobre gobiernos locales. Varias cuestiones quedan pendientes en la agenda de investigación: la permanencia de las innovaciones, el tipo de participación ciudadana estimulada, la perdurabilidad de las alianzas, o bien el carácter «minimalista» de la de- 
mocracia local, en contextos globales y nacionales de economías neoliberales (Goldfrank, 20I6: 72-76).

Desde este panorama general, cabe preguntarse cuáles han sido las lecturas de la teoría del régimen desde países latinoamericanos, digamos, con niveles de desarrollo económico más adelantados (Brasil, México, Argentina, Chile, Colombia).

Serán necesarios diálogos más fructíferos entre comunidades académicas para intentar romper los etnocentrismos que la tipifican. Ayudarían una mayor discusión de conceptos, teorías y experiencias a contrastar y reevaluar, desde los espacios urbanos situados en diferentes órbitas del capitalismo global. En tal sentido, una perspectiva relacional que atienda las emergencias y las ausencias (aquellos factores no visualizados en las propuestas teóricas dominantes) podría ayudar a superar la relación más bien fantasmal entre teoría y prácticas sociales en el espacio urbano contemporáneo.

La perspectiva de análisis del régimen urbano puede ser atendida desde el Sur (a partir de México) y para el Sur (América Latina, el Caribe), pero en diálogo franco con los teóricos de la fórmula original y sus posteriores desarrollos. Una teoría regional sobre el régimen urbano podría ayudar a descifrar esos códigos ocultos, bajo los cuales se ha construido la ciudad latinoamericana. De ahí el discreto encanto que la envuelve, aun cuando en ocasiones predomine la bruma y todo parezca indicar que (¿erróneamente?) ya es hora de dejarla en paz. Una ecología de saberes, por sí misma diversa, plural y polifacética, haría visibles procesos y dimensiones desconocidas sobre los procesos de producción del hábitat, y podría distinguir y clasificar algunas de las expresiones del poder, acorde a sus correspondientes escalas espaciales.

\section{REFERENCIAS}

Aglietta, M. (1999). Regulación y crisis del capitalismo. México: Siglo XXI. Bassols, M. (1994). Reforma urbana y poder. El escenario estadounidense. En Bassols, M. (coord.), Campo y ciudad en una era de transición. Problemas, tendencias y desafíos. México: Departamento de Sociología, Universidad Autónoma Metropolitana-Iztapalapa. 
Bassols, M. (coord., 2006). Explorando el régimen urbano en México. Un análisis metropolitano. México: El Colegio de la Frontera Norte, Universidad Autónoma Metropolitana-Iztapalapa, Plaza y Valdés.

Carrión, F. y Ponce P. (coords., 2015). El giro a la izquierda: los gobiernos locales de América Latina. $2^{\mathrm{a}}$ reimpresión ( $5^{\mathrm{a}}$ ed.). México: Avenida.

Dahl, R. (196I). Who Governs? Yale University Press: New Haven.

Davies, J. (2002). Urban Regime Theory: a Normative-Empirical Critique. Journal of Urban Affairs. Urban Affairs Association, 24 (I), I-I7.

Domhoff, W. (202I). Atlanta: Floyd Hunter Was Right. Who Rules America? <www.whorulesamerica.ucsc.edu/local/atlanta.html>.

Duhau, E. (2006). Introducción. En Bassols, M. (coord.). Explorando el régimen urbano en México. Un análisis metropolitano. México: El Colegio de la Frontera Norte, Universidad Autónoma Metropolitana-Iztapalapa, Plaza y Valdés.

Forrest, R. y Wissink, B. (20I7). Whose City Now? Urban managerialism reconsidered (again). The Sociological Review, 65(2), I55-I67.

García, C. (2016). Teorías e historia de la ciudad contemporánea. Barcelona: Gustavo Gili.

Goldfrank, B. (20I5). Una Mirada Panorámica a la Primera Ola Local de la Izquierda. En Carrión, F. y P. Ponce (coords.). El giro a la izquierda: los gobiernos locales de América Latina. México: Avenida, 57-79.

Guarneros, V. (2006). Los pros y los contras desde una perspectiva europea. En Bassols, M. (coord.). Explorando el régimen urbano en México. Un análisis metropolitano. México: El Colegio de la Frontera Norte, Universidad Autónoma Metropolitana-Iztapalapa, Plaza y Valdés.

Guevara, T. (2015). ¿La ciudad para quién? Transformaciones territoriales, políticas urbanas y procesos de producción del hábitat en la ciudad de Buenos Aires (1996-20II). México: Coordinación de Humanidades-Unam, PUEC-Unam, Infonavit. 
Hankins, K. (20I5). Regime Politics in Geography. Urban Affairs Review, $S A G E$, vol. SI(I), I50-I60.

Hernández, A. (2008). De la dialéctica a la trialéctica del espacio: aproximaciones al pensamiento de Milton Santos y Edward Soja. En Mendoza, C.1: (coord.). Tras las huellas de Milton Santos. Una mirada latinoamericana a la geografía contemporánea. Barcelona: Anthropos, 84-97.

Jones-Correa, M. and Wong D. (2015). Whose Politics? Reflections on Clarence Stone's. Regime Politics. Urban Affairs Review, SAGE, SI (I), I6I-I70.

Lauria, M. (ed., 1997). Reconstructing Urban Regime Theory. Regulating Urban Politics in a Global Economy. London, New Delhi: SAGE, Thousand Oaks.

Mayer, M. (20I7). Whose City? From Ray Pahl's Critique of the Keynesian city to the Contestations Around Neoliberal Urbanism. The Sociological Review, SAgE, 65(2), I68-183.

Merino, M. (2013). Políticas públicas. México. Ensayo sobre la intervención del Estado en la solución de problemas públicos. Centro de Investigación y Docencia Económica.

Molotch, H. (1976). The City as a Growth Machine. American Journal of Sociology, (82), 309-330.

Mollenkopf, J. (1983). The Contested City, Princeton University Press: Princeton.

Mossberger, K. y Stoker G. (200I). The Evolution of Urban Regime Theory. Urban Affairs Review, 36(6).

Peterson, P. (198I). City Limits, Chicago: University of Chicago.

Porras, F. (20I6). Gobernanza. Propuestas, limites y perspectivas. México: Instituto Mora.

Rast, J. (2015). Urban Regime Theory and the Problem of Change. Urban Affairs Review, SAGE, SI(I), I38-I49.

Stoker, G. (1995). Regime Theory and Urban Politics. In Judge, D., Stoker, G. and H. Wolman (eds.). Theories of Urban Politics. London: SAGE.

Stone, C. (1989). Regime Politics. Governing Atlanta, 1946-1988. Kansas: Press of Kansas, Lawrence. 
Stone, C. (1993). Urban Regimes and the Capacity to Govern: a political Economy Approach. Journal of Urban Affairs. SAGE (I5), I-28. Stone, C. (200I). The Atlanta Experience Re-examined. International Journal of Urban and Regional Research (25) 20-34.

Stone, C. (2005). Looking Back to Look Forward: Reflections on Urban Regime Analysis. Urban Affairs Review (40), 309-34I.

Stone, C. (2008). Rethinking the Policy-Politics Connection. En Jonathan Davies (ed.). Innovations in Urban Politics. New York: Routledge Stone, C. (2015). Reflections on Regime Politics: from Governing Coalition to Urban Political Order. Urban Affairs Review sage. SI(I), IOI-I37.

Tilly, Ch. (1984). Big Structures, Large Processes, Huge Comparisons. New York: Russell Sage Foundation. 